\title{
Studies on Ejection of Cell Culture Medium by Electrohydrodynamic Method
}

\author{
Zhang Haiyi ${ }^{1}$, Wang $\mathrm{Can}^{2}$, Wang $\mathrm{Fei}^{1}$, Wang Yiwei ${ }^{1}$, Wang Zhihai ${ }^{1}{ }^{*}$, , Chen $\mathrm{Xi}^{3}$, and Gui Jingang ${ }^{3}$ \\ ${ }^{1}$ Faculty of Information Technology, Beijing University of Technology, Beijing 100124, China \\ ${ }^{2}$ Laser Engineering Research Institute, Beijing University of Technology, Beijing 100124, China \\ ${ }^{3}$ Beijing Institute of Pediatrics, Beijing Children's Hospital, Capital Medical University, Beijing 100035, China
}

\begin{abstract}
Sample deposition based on micro-droplet ejection has broad application prospects in the field of biomedicine. Ejection of RPMI-1640 medium (without and with cells) is investigated experimentally using a home-build electrohydrodynamic (EHD) ejection system, consisting of a liquid supplier and a nozzle, a high voltage source, a droplet collector, and a high speed photography module. Electric voltage is applied between the nozzle and the droplet collector. The liquid surface is charged and the ejection takes place when electric force overcomes the surface tension. The ejection process is studied by using high speed photography and image processing. At low voltage, a uniform ejection state is established with ejection frequency typically less than $50 \mathrm{Hertz}$. At sufficiently high voltage, another uniform ejection state is reached with ejection frequency as high as $1300 \mathrm{~Hz}$. Human peripheral blood mononuclear cells, after ejection, show survival rates higher than $79 \%$, manifesting EHD ejection as a promising technique for cell printing.
\end{abstract}

\section{Introduction}

The micro-droplet ejection technology has been extended from the popular inkjet printing field to many scientific and technical fields such as fabrication of micro/nano structures, microelectronic packaging, and genetic engineering [1]. Especially in the field of biomedicine, micro-droplet ejection technology is suitable for a wide range of "bio-inks", with cells, proteins, DNAs, and other bio-active materials included [2]. In the past 20 years, the rapid development of drug screening, sustained release technology, tissue engineering, cell assembly has opened up new broad application prospects for precise deposition of biological samples based on micro-droplet ejection technology

At present, typical micro-droplet ejection utilizes thermal, piezoelectric, pneumatic, or electrostatic techniques [1]. For the thermal ejection, a heating element quickly evaporates the liquid in a local region, forming a bubble, which rapidly expands, generates a pressure pulse in the liquid storage chamber. The pressure pulse squeezes the liquid through a nozzle, overcomes the surface tension to forms a micro-droplet. For biomedical applications, transient high temperatures can have negative impacts on some biological properties. Both piezoelectric and electrostatic micro-droplet ejections use voltage pulses to actuate a deformation (piezoelectric deformation or electrostatic deformation) of the liquid storage chamber, squeezing the liquid out of the nozzle to produce the micro-droplets. For the pneumatic micro-droplet ejection, the pressure pulse in the liquid chamber is generated via a high pressure gas source under control of a high speed solenoid valve. For the micro-droplet ejection, it is advantageous to reduce the size of the droplets for higher resolution in material deposition. Although all the above mentioned droplet ejection technique can be used for bio-printing, those techniques rely on squeezing the liquid through the nozzle, and the resulting droplet diameter is usually larger than the nozzle diameter [3]. If smaller droplets are required, reducing the nozzle size is the most direct and effective approach. However, reducing the nozzle diameter greatly increases the required driving pressure, and leads to more damage to the "bio-inks" (such as cellladen liquids). Moreover, smaller nozzle is prone to clog, especially for "bio-inks" containing cells or other biological suspensions. In recent years, although a large number of studies have attempted to generate droplets smaller than the nozzle diameter [3] by applying more complicated driving pulse waveforms, those techniques are still in the research stage, and no extensive engineering applications have been realized.

Unlike the traditional method of "pushing" (or "squeezing"), electrohydrodynamic (EHD) micro-droplet ejection, by "pulling" the liquid at the nozzle via electric force, attracted a lot of interests [4]. As shown in Fig. 1(a), electric voltage is applied between the nozzle and a grounding electrode to create an electric field. The liquid at the nozzle is electrically charged, and therefore experienced electric force in the field. Some typical ejection modes, referred as dripping mode, microdripping mode, cone-jet mode, spindle mode, and multijet mode [5], are shown in Fig. 1 (b) - (f). At sufficiently low voltage, the effects of the electric force are

* Corresponding author: wangzhihai@ bjut.edu.cn 
negligible, the liquid at the nozzle exhibits a hemispherical or ellipsoid meniscus due to interplay of the gravity and the surface tension (Fig. 1(b)). The droplets generated are typically larger than the outer diameter of the nozzle, and to the order of millimeter in size. Gradually increasing the voltage, the electric force becomes more relevant, and the ejection evolves into the micro-dripping mode (Fig. 1(c)). The liquid forms a cone shape meniscus (Taylor cone) [6]. The end tip of the liquid cone breaks up to form a droplet, and the droplet can be much smaller than the diameter of the nozzle. This greatly alleviates the problem of nozzle clogging. The ejection frequency is from several Hertz to several kilo-Hertz depending on the voltage, on the flow rate, and also on hydrodynamic and electric properties of the liquid. The cone-jet mode (Fig. 1(d)) usually occurs at higher flow rate. Due to Varicose instability, the jet breaks into droplets, and the frequency of micro-droplet generation is usually from several kilo-Hertz to several mega-Hertz. For those three modes, monodispersed droplets are produced. At even higher voltages, the spindle mode and the multi-jet mode can occur. Those ejection modes result in non-monodispersed microdroplets, and are typically difficult to control.

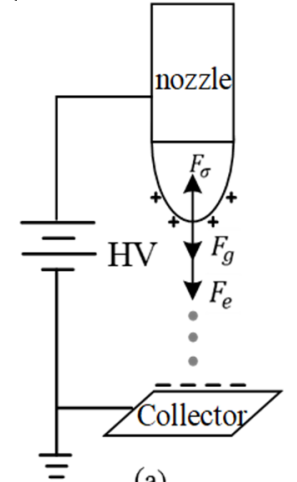

(a)

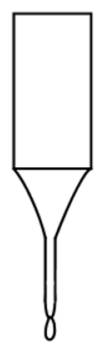

$\circ$

(d)
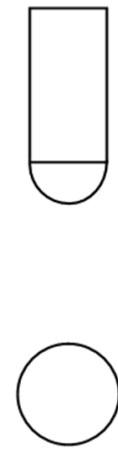

(b)

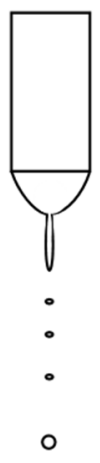

(e)

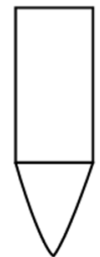

○

(c)

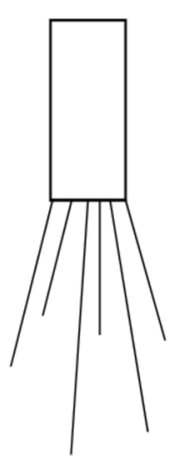

(f)
Fig. 1. Basic principles of electrohydrodynamic droplet ejection. Main function modes of the EHD ejection.

(b)Dripping mode, (c) Micro-dripping mode, (d) Cone-jet mode, (e) Spindle mode, (f) Multi-jet mode

EHD printing is suitable for a wide range of materials, including "inks" containing insulating or conductive polymers, metal nanoparticles, nanowires or carbon nanotubes [4]. In recent years, experimental groups have used EHD droplet ejection technology for biological cell printing [7]. It should be noted that sizes of typical cells range from several microns to several tens of microns. If the cells are to be encapsulated, droplets larger in size are required. In the micro-dripping mode, both the electric stress and the ejection frequency are moderate, monodispersed droplets with size in the range of several tens of microns to several hundred microns can be generated. This mode is therefore suitable for the printing of biological cells

\section{Experiments and methods}

The home-build EHD micro-droplet ejection system mainly comprises a liquid supply device connected with a stainless steel nozzle, a micro-droplet collector, a high voltage (HV) power supply, and a high speed photography module, as shown in Fig. 2.

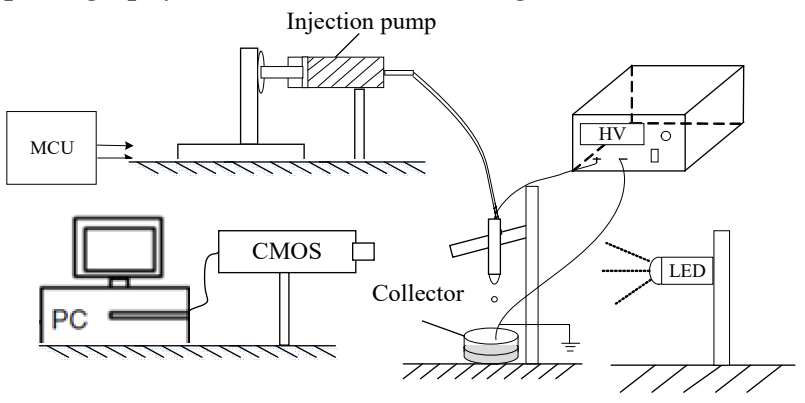

Fig. 2. Schematic of a home-built EHD micro-droplet ejection system, consisting of a syringe pump based liquid supplier, a nozzle, a high voltage (HV) source, a micro-droplet collector, and a high speed camera.

The liquid supplier is based on a syringe pump, modified from a linear electric stage (KSA100-11 from Zhuoli Hanguang), with a planetary gear reducer of 1:100 for improving uniformity of the liquid flow. The stepper motor driver supports $1 / 128$ micro-stepping. The ball screw has a lead of $4 \mathrm{~mm}$. Using a $5 \mathrm{ml}$ syringe, the syringe pump can provide flow rate from $10 \mu \mathrm{L} / \mathrm{h}$ to $800 \mu \mathrm{L} / \mathrm{h}$. The syringe pump is connected to a stainless steel nozzle with an inner diameter of $0.13 \mathrm{~mm}$ and an outer diameter of $0.31 \mathrm{~mm}$.

For collection of the micro-droplets, a culture dish (30mm in diameter) containing phosphate buffered saline (PBS) is placed $10 \mathrm{~mm}$ below the nozzle, and is connected to the electric grounding pole of the whole ejection system. The design of the micro-droplet collector is based on following considerations. The ejected liquid would disperse into the PBS in the culture dish, avoiding the phenomenon of liquid accumulation, which would modify the electric field distribution as the ejection continues. In addition, PBS not only exhibits good electric conductivity to quickly release electric change carried by the droplet, but also helps to maintain the viability of cells encapsulated in the micro-droplet (see subsection 3.2).

The adjustable high-voltage is provided by using a DC high-voltage power supply (Dongwen High-voltage Power Supply Co., Tianjin, model DW-P503-1ACDF), with output voltage range $0-50 \mathrm{kV}$, and maximum output current up to $1 \mathrm{~mA}$. In the experiment, the positive electrode is connected to the stainless steel nozzle, and the negative electrode is connected to the ground pole of the entire ejection system. 
For recording the process of the droplet generation, a photography module, consisting mainly of a highbrightness LED and an ultra-high speed camera (Keyence's VW-600C, with VH-Z20R zoom lenses also from Keyence), is incorporated. Photographing is done by using back-lightening configuration (see Fig. 2), with LED brightness kept constant. Frame rate is set to 8000 frames/second, and the exposure time per frame is $1 / 70000$ seconds. The speed of the droplet can be estimated as $2-4 \mathrm{~m} / \mathrm{s}$, so the droplet displacement during the exposure time is about $30-60 \mu \mathrm{m}$. For smaller microdroplets, the shadow is comparable to the droplet (see Fig.4., for example). In the future, the above shortcomings can be solved by using ultra-short exposure based on stroboscopic lamps.

The ultra-high speed camera generates a large amount of image data. In order to better display those data, the original image is sequentially processed by a commercial image processing software package (with MATLAB). A region of interest (ROI), much smaller than the raw image, is defined to enhance the image processing speed. It is followed by a morphological closed operation for lower noise, and an automatic threshold segmentation for improving contrast. The meniscus is measured based on the "Sobel" edge detection operator, and then basic geometrical information of the meniscus can be extracted. Specifically in this paper, the time dependent length of the meniscus, in the axial direction, is an important measure of the droplet ejection process, and will be displayed in the following section.

\section{Experimental results and discussions}

The liquid to be ejected in the experiment is mammalian cell medium RPMI-1640. The main composition of RPMI-1640 is balanced salt solution, supplemented with amino acids, vitamins and other nutrients similar to those in serum. The viscosity coefficient of RPMI-1640 medium is $2 \times 10^{-3} \mathrm{~Pa} \cdot \mathrm{s}$ [8].

\subsection{EHD ejection of pure RPMI-1640 medium}

The flow rate is maintained at $200 \mu \mathrm{L} / \mathrm{h}$. The ejection states are studied at several voltages. When the voltage is lower than about $3 \mathrm{kV}$, the droplet diameter is much larger than the outer diameter of the nozzle $(0.3 \mathrm{~mm})$, and is up to $1-2 \mathrm{~mm}$. The droplet generation frequency is less than $1 \mathrm{~Hz}$. The ejection is working in the dripping-mode (see Fig. 1(b)).

Above about $3 \mathrm{kV}$, the ejection becomes microdripping, marked by the appearance of cone-shape meniscus. As the voltage is increased, the droplet diameter gradually decreases and the ejection frequency increases.

A typical ejection state is shown in Fig. 3, where the voltage is $\mathrm{U}=3.3 \mathrm{kV}$. The ejection is stable versus time. The droplet size is about $130 \mu \mathrm{m}$. As shown in Fig. 3(b), the ejection frequency is about $48 \mathrm{~Hz}$. For each droplet ejection, the length of the meniscus shows a slow linear growth, followed by sharp peak that is associated with the meniscus breakup. This stable ejection state is referred as state I.

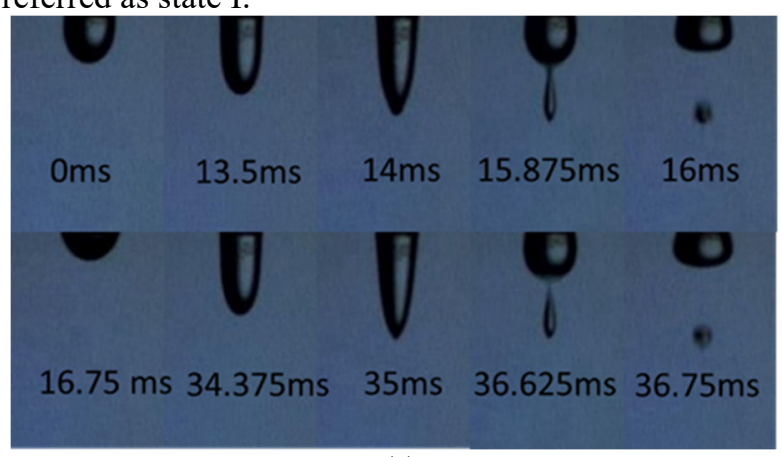

(a)

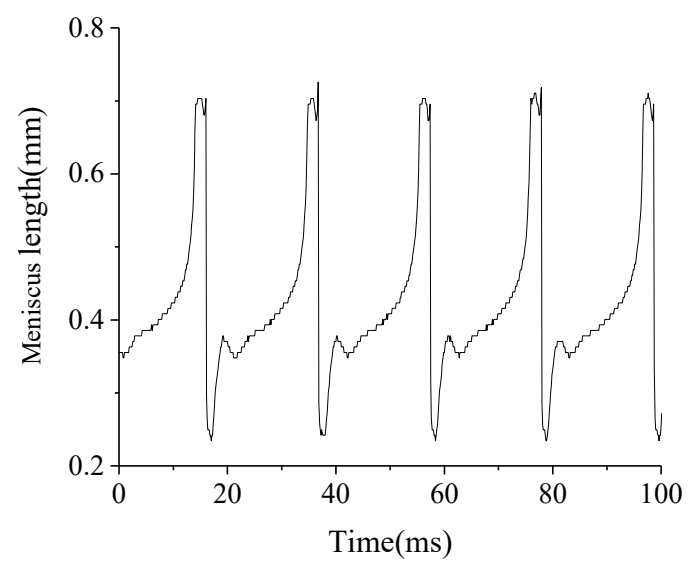

(b)

Fig. 3. (a) Ejection state I, for voltage $U=3.3 \mathrm{kV}$. (b) Time dependence of the meniscus length at the tip of the nozzle, along the axis of the nozzle.

At sufficiently high voltage, the ejection stays in another stable state II. As shown in Fig. 4, for $\mathrm{U}=3.6 \mathrm{kV}$, the droplet size is uniform, the size is about $70 \mu \mathrm{m}$, and the ejection frequency can reach about $300 \mathrm{~Hz}$. Keeping increasing voltage to some extend tends to slightly increase this ejection frequency, but with the tuning range typically less than $20 \%$. It should be mentioned here that some different ejection frequency ranges (ex. $600-700 \mathrm{~Hz}$ and $1000-1200 \mathrm{~Hz}$ ) are also observed, but typically from some different initial conditions. At all those situations, the ejection states are stable, and the droplet sizes are uniform.

Not shown in this paper, the ejection states at intermediate voltages are not stable, and therefore are not relevant to cell printing described in the next subsection.

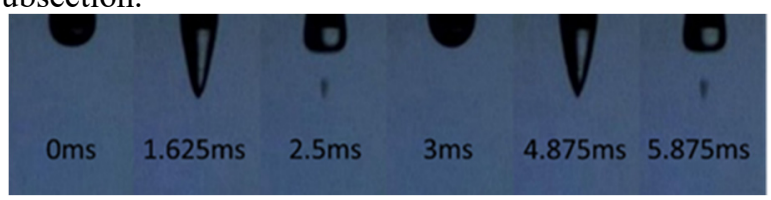

(a) 


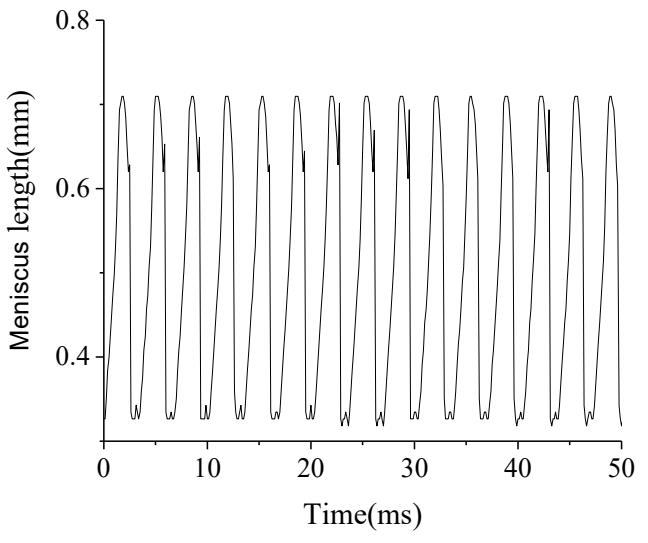

(b)

Fig. 4. (a) Ejection state II, for voltage $U=3.6 \mathrm{kV}$. (b) Time dependence of the meniscus length at the tip of the nozzle, along the axis of the nozzle

\subsection{Ejection of cell-laden RPMI-1640}

After the ejection experiment of pure RPMI-1640 medium, ejection of RPMI-1640 containing human peripheral blood mononuclear cells (PBMC) are performed, and the effects of the ejection process on cell viability are evaluated. PBMC are separated from human peripheral blood by density gradient centrifugation. The cell concentration is about $10^{6} / \mathrm{mL}$. As described above, cell-laden RPMI-1640 is ejected into a culture dish containing PBS $(0.5 \mathrm{~mL})$.

For survival rate evaluation, two samples (A and $\mathrm{B}$ ) are collected from two ejection states similar to the states I and II. Sample A is taken with voltage set to $3.2 \mathrm{kV}$, based on data from the photographic module, the cell ejection state is stable, the ejection frequency is $20 \mathrm{~Hz}$, the droplet diameter is about $170-180 \mu \mathrm{m}$, and is uniform. Sample B is taken at the voltage $3.61 \mathrm{kV}$. The ejection frequency is $1.3 \mathrm{kHz}$, the droplet diameter is about $45 \mu \mathrm{m}$, and the droplet size is also uniform. In addition, $60 \mu \mathrm{L}$ of the PBMC suspension is directly squeezed through the nozzle into the PBS $(0.5 \mathrm{~mL})$ without applying electric voltage, producing sample $\mathrm{C}$ that is labelled as the control group.

For each sample, $1 \mu \mathrm{L}$ of 7-AAD (7-aminoactionmycin D) is added for fluorescent antibody staining, and after 15 minutes of staining, flow cytometry is used for evaluating cell viability based on the fluorescence expression intensity. (a)
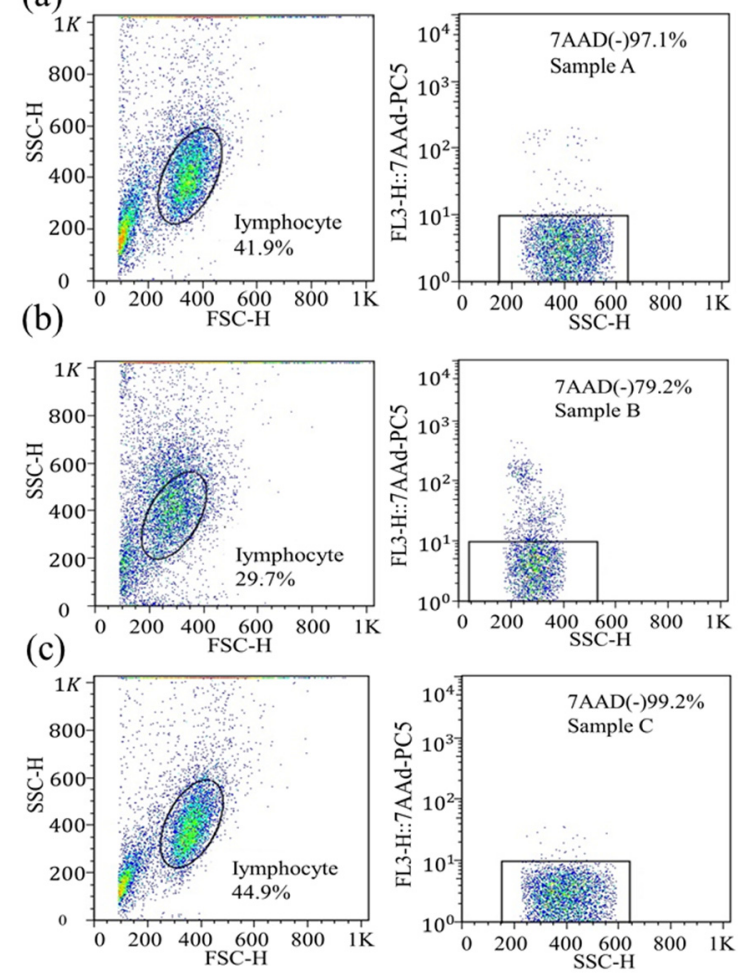

Fig. 5. The survival rates of cells are evaluated by flow cytometry using 7-AAD staining. (a) Sample A (Ejection state I), for voltage $U=3.2 \mathrm{kV}$. (b) Sample B (Ejection state II) for voltage $\mathrm{U}=3.61 \mathrm{kV}$. (c) Sample C, the control group (PBMC suspension directly squeezing through the nozzle).

As shown in the figure, FSC-H and SSC-H in left panels are intensities of forward scattered light and side scattered light, respectively, and the data points in the elliptical gate correspond to PBMC. The proportion of PBMC in samples $\mathrm{A}$ and $\mathrm{C}$ are $41.9 \%$ and $44.9 \%$ respectively. Their differences are moderate and reasonable. The proportion of PBMC in sample B is $29.7 \%$, greatly reduced.

For right panels of Fig. 5, the vertical axis denotes the relative fluorescence intensities of the 7AAD fluorescent dyes, and the gated data points denote cells whose $7 \mathrm{AAD}$ are negative $(7 \mathrm{AAD}(-))$, i.e. are still viable. It can be seen from the figure that the survival rate of PBMC in the control group (sample C) is $99.2 \%$, and the PBMC viabilities for samples $\mathrm{A}$ and $\mathrm{B}$ are $97.1 \%$ and $79.2 \%$, respectively. It is suggested that the high frequency ejection (state II) clearly deteriorates the cell viability.

For samples A (from ejection states I), the cells are encapsulated in a set of rather large droplets, with diameter around $170 \mu \mathrm{m}$ (see Fig. 3). The sheer force exerting on the cells might still be moderate. For sample B (from ejection state II), the cells are encapsulated in much smaller droplets (around $45 \mu \mathrm{m}$ in diameter). The sheer force on the cells is expected to be much stronger. It has been found that the sheer force during the microdroplet generation has negative impacts on the cell viability [10-11]. More quantitative estimation of sheer force might help the interpretation of the viability data. 


\section{Conclusions}

This paper introduces a home-build EHD micro-droplet ejection system and describes the ejection of the RPMI1640 medium, without and with cells. As the main purpose of this study is to evaluate EHD ejection as possible technique for cell printing, we focus on the micro-dripping mode. Experiments have shown that the ejection state depends on the voltage when other parameters, such as flow rate and geometrical parameters, are fixed. When the voltage is low, for ejection state I, the droplets produced are uniform in size, and on increasing voltage the ejection frequency increases from a few Hertz to a few tens of Hertz. At sufficiently high voltage, in state II, the ejection reaches another uniform state, with ejection frequency much higher. The corresponding droplet size is largely reduced, compared with that for state I.

The RPMI-1640 medium containing human PBMC is ejected. In the case of ejection state $\mathrm{I}$, the ejection process has negligible effect on the cell viability, while for state II, the number of cells decrease after the ejection, and the cell viability deteriorates. For state II, droplet size is much smaller, and the droplet generation process is much quicker. The stronger sheer force associated with the ejection might be the cause of the cell damages. Although high frequency ejections result in decreased cell viability, the survival rate is still close to $80 \%$, suggesting that the EHD ejection is a promising technical option for the cell printing.

\section{References}

1. W. Zapka, Handbook of Industrial Inkjet Printing: A Full System Approach, Wiley-VCH (2018)

2. H. Gudapati, M. Dey, I. Ozbolat, A Comprehensive Review on Droplet-based Bioprinting: Past, Present and Future, Biomaterials, 102, PP20-42 (2016)

3. A. U. Chen, and O. A. Basaran, A new method for significantly reducing drop radius without reducing nozzle radius in drop-on-demand drop production, Phys. Fluids, 14, L1 (2002)

4. M. S. Onses, E. Sutanto, P. M. Ferreira, A. G. Alleyne, and J. A. Rogers,Mechanisms, Capabilities, and Applications of High-Resolution Electrohydrodynamic Jet Printing, Small, 11, PP4237-4266 (2015)

5. M. Cloupeau and B. Prunet-Foch, Electrohydrodynamic Spraying Functioning Modes: A Critical Review, J. Aerosol Sci., 25, PP1021-1036 (1994)

6. G. Taylor, Disintegration of Water Drops in an Electric Field, Proc. R. Soc. A, 280, PP383 - 397 (1968)

7. V. L. Workman, L. B. Tezera, P. T. Elkington, and S. N. Jayasinghe, Controlled Generation of Microspheres Incorporating Extracellular Matrix Fibrils for Three-Dimensional Cell Culture, Adv. Funct. Mater., 24, PP2648 - 2657 (2014)
8. M. Zou, S. Cai, Z. Zhao, L. Chen, Y. Zhao, X. Fan, and S. Chen, A novel polydimethylsiloxane microfluidic viscometer fabricated using microwiremolding, Rev. SCI. Instrum., 86, 104302 (2015)

9. P. Galliker, J. schneider, H. Eghlidi, S. Kress, V. sandoghdar, D. Poulikakos, Direct printing of nanostructures by electrostatic autofocussing of ink nanodroplets, Nat. Commun, 3(2), PP890-899 (2012)

10. A. Blaeser, D. F. Duarte Campos, U. Puster, W. Richtering, . M. Stevens, and H. Fischer, Controlling Shear Stress in 3D Bioprinting is a Key Factor to Balance Printing Resolution and Stem Cell Integrity, Adv. Healthc. Mater., 5(3), PP326 - 333 (2016)

11. E. Cheng, H. Yu, A. Ahmadi, and K. C. Cheung, Investigation of the hydrodynamic response of cells in drop on demand piezoelectric inkjet nozzles, Biofabrication, 8, 015008 (2016) 